\title{
ESTRÉS PERCIBIDO EN TRABAJADORES DE ATENCIÓN PRIMARIA ${ }^{1}$
}

\section{PERCEIVED STRESS AMONG PRIMARY HEALTH \\ CARE WORKERS}

\section{ESTRESSE PERCEBIDO EM TRABALHADORES DE ATENÇÃO PRIMÁRIA}

\author{
Paula Ceballos-Vásquez* \\ José Solorza-Aburto** \\ Nicol Marín-Rosas*** \\ JAQUEline MoragA**** \\ Nataly GómeZ-Aguilera ${ }^{* * * * *}$ \\ Francés Segura-ArRiagadA ${ }^{* * * * * *}$ \\ RafaEla Andolhe*******
}

\begin{abstract}
RESUMEN
Introducción: El estrés laboral es considerado una epidemia, siendo el único riesgo ocupacional que puede afectar la salud de todos los trabajadores. Objetivo: Evaluar la percepción de estrés del equipo de enfermería de Atención Primaria en Salud de una comuna de la Región del Maule-Chile relacionándolo con el perfil sociodemográfico, variables laborales y condiciones ambientales de trabajo. Material y método: Diseño transversal, de abordaje cuantitativo. Participaron 105 trabajadores, enfermeros/as y técnicos de enfermería de APS en una comuna del centro sur de Chile. Se recolectaron datos con instrumento que incluyó: "Antecedentes biosociodemográficos" y "Job Content Questionnaire (JCQ)". Los datos se analizaron con Correlación de Pearson y Chi-cuadrado. La investigación contó con la aprobación de los Comités Ético-Científico de la Universidad Católica del Maule y del Servicio de Salud del Maule. Durante toda la investigación se contemplaron los principios éticos de E. Emanuel. Resultados: El 23,6\% de los participantes percibió alto nivel de estrés. Existen relaciones estadísticamente significativas $(p \leq 0,05)$ entre variables biosociodemográficas (edad y número de hijos), variables laborales (cargo y experiencia en años) y con las condiciones ambientales de trabajo (iluminación, temperatura, ruido, entre otras). Conclusiones: Los trabajadores del equipo de
\end{abstract}

\footnotetext{
1 Trabajo derivado de la Tesis "Percepción de estrés en el equipo de enfermería de atención primaria en salud". Programa de Licenciatura en Enfermería, Universidad Católica del Maule, Sede Talca. Chile.

* Enfermera. Departamento de Enfermería, Facultad de Ciencias de la Salud. Universidad Católica del Maule. Miembro del Centro de Investigación del Cuidado UC del Maule. Chile. Email: pceballos@ucm.cl. Autor de correspondencia.

** Licenciado en Enfermería. Escuela de Enfermería, Universidad Católica del Maule, Sede Talca, Chile.

Email: jsolorzaa@gmail.com

*** Enfermera. Escuela de Enfermería, Universidad Católica del Maule, Sede Talca. Chile. Email: nicolmarinro@gmail.com

**** Enfermera. Escuela de Enfermería, Universidad Católica del Maule, Sede Talca. Chile. Email: moraga.alejandra6@gmail.com

*****Estudiante de Enfermería. Escuela de Enfermería, Universidad Católica del Maule, Sede Talca, Chile.

Email: natalygomez196@gmail.com

****** Estudiante de Enfermería. Escuela de Enfermería, Universidad Católica del Maule, Sede Talca. Chile.

Email: francesdelr@gmail.com

******* Enfermera. Departamento de Enfermería. Universidad Federal de Santa María, RS, Brasil. Email: rafaela.andolhe@ufsm.br
} 
enfermería de Atención primaria, perciben estrés al desempeñar su trabajo. Además, se estableció una relación estadística entre estrés y condiciones ambientales de trabajo, por lo que se invita a las organizaciones a enfatizar en la fiscalización y supervisión, para de reducir el porcentaje de trabajadores en riesgo de alterar su salud a causa del estrés laboral.

Palabras clave: Riesgos laborales; Estrés; Atención Primaria de Salud; Enfermería.

\section{ABSTRACT}

Introduction: Work-related stress is considered a global epidemic, being the only occupational risk that can affect the health of $100 \%$ of workers. Objective: To evaluate the level of stress perceived by nurses in Primary Health Care, based on the socio-demographic profile, work variables and work environmental conditions. Materials and methods: Quantitative, cross-sectional study with 105 Primary Health Care workers, nurses and nursing technicians of a city in south central Chile. Data were collected with an instrument divided in: "Biosociodemographic background" and "Job Content Questionnaire (JCQ)". The data were analyzed using Pearson correlation and chi-square. The research was approved by the Committee on Scientific Ethics of the Universidad Católica del Maule and the Health Service of the Maule Region. The ethical principles of E. Emanuel were considered throughout the study. Results: $23.6 \%$ of participants perceived a high level of stress. There are statistically significant relations $(p \leq 0.05)$ between biosociodemographic variables (age and number of children), work variables (position and experience in years) and work environmental conditions (lighting, temp erature, noise, among others). Conclusions: Workers from the nursing team perceive stress while doing their job. In addition, a statistical relationship can be established between stress and work environment. Organizations are therefore encouraged to supervise the work environment in order to reduce the percentage of workers at risk of altering their health due to work stress.

Key words: Occupational risks; Stress; Primary Health Care; Nursing.

\section{RESUMO}

Introdução: $\mathrm{O}$ estresse laboral é considerado uma epidemia global, sendo o único risco ocupacional que pode afetar a saúde de todos os trabalhadores. Objetivo: Avaliar o nível de estresse percebido pela equipe de enfermagem de Atenção Primária à Saúde (APS) e associação com o perfil sociodemográfico e laboral, bem como às condiçóes ambientais de trabalho. Material e método: Estudo transversal, com abordagem quantitativa. Participaram 105 trabalhadores de enfermagem (enfermeiros e técnicos) em um distrito chileno. Para a coleta de dados foi utilizado instrumento dividido em: antescedentes biosociodemográficos e o Job Content Questionnaire (JCQ). Para a análise das associaçôes foram utilizados os teste Qui-Quadrado e Correlação de Pearson. A pesquisa foi aprovada por Comitê de Ética da Universidade Católica do Maule e do Serviço de Saúde de Maule, respeitando os princípios éticos de E. Emanuel. Resultados: 23,6\% dos participantes perceberam alto nível de estresse. Existem relações estatisticamente significativas $(p \leq 0,05)$ entre variáveis biosociodemográficas (idade e número de filhos), variáveis laborais (cargo e experiência) e entre as condições ambientais de trabalho (iluminação, temperatura, ruído, entre outros). Conclusôes: Concluiu-se que os trabalhadores de enfermagem da APS percebem o estresse ao desempenhar seu trabalho. Além disso, houve associação estatisticamente significativa entre estresse e condiçôes do ambiente de trabalho, o que justifica as instituições atentarem para a fiscalização e supervisão no trabalho, com vistas reduzir a prevalência de trabalhadores com risco de comprometer sua saúde em decorrência do estresse laboral.

Palavras chave: Riscos Ocupacionais; Estresse Psicológico; Atenção Primária à Saúde; Enfermagem do Trabalho. 


\section{INTRODUCCIÓN}

A nivel mundial, el estrés laboral es considerado como una epidemia global, siendo el único riesgo ocupacional que puede afectar al cien por ciento de los trabajadores/as. Expertos puntualizan que podría generar ausentismo laboral, disminución de la productividad y del rendimiento individual, lo que podría generar alteraciones del estado de salud, aumento de enfermedades y accidentes en los trabajadores/as ${ }^{(1-3)}$. Lo expuesto a nivel mundial no dista de lo que sucede en Chile. De hecho, la Asociación Chilena de Seguridad (ACHS) indica que el $48 \%$ de las licencias médicas son por causa de enfermedades profesionales asociadas a problemas de la salud mental, entre ellos, el estrés $^{(4)}$. Dicho riesgo psicosocial se encuentra presente en todas las profesiones, sin embargo, los trabajadores sanitarios, como el equipo de enfermería de Atención Primaria en Salud (APS), está expuesto en mayor medida por su labor de entrega de cuidados, interacción con otros seres humanos, numerosas interrupciones como llamadas telefónicas, ingreso reiterado de personas al box de atención, ruidos provenientes de la sala de espera, entre otros aspectos, lo que pudiese generar distrés o tensión mental y, finalmente, causar enfermedades.

Autores indican que el estrés pudiera generar alteraciones en la salud tanto física y mental en los trabajadores del área de asistencia -específicamente en el área de enfermería-, la cual es considerada como una de las profesiones estresantes a nivel mundial ${ }^{(5)}$. El Consejo Internacional de Enfermeras (CIE) indica que es improbable que las profesionales de Enfermería puedan desarrollarse óptimamente y alcanzar los resultados deseados en términos de salud, bajo la tensión que generan los entornos de trabajo ${ }^{(6,7)}$.

Se puntualiza que este contexto laboral no ha sido estudiado en todos los niveles de atención de salud, ya que se ha prestado principal atención a las manifestaciones de estrés en los profesionales que se desempeñan en el área intrahospitalaria, quedando ampliamente expuesto en la literatura ${ }^{(8,9)}$. A partir de esto se desprende el problema de investigación, ya que se desconoce el nivel de estrés percibido por el equipo de Enfermería de Atención Primaria en Salud en una comuna de la Región del Maule.

Para el abordaje de este estudio se utilizaron como soportes teóricos, el modelo de DemandaControl y Apoyo Social postulado por Karasek y Theorell ${ }^{(10)}$, el que intenta describir y analizar situaciones laborales estresantes y pone énfasis en las características psicosociales del entorno del trabajo. Este ha sido sin lugar a dudas uno de los modelos más influyentes en las investigaciones sobre riesgos psicosociales en el trabajo, estrés y enfermedad desde principios de los años ochenta $^{(11)}$. Sumado a la teoría de consecución de metas de Imogene $\operatorname{King}^{(12,13)}$, la cual responde y define conceptos centrales para este estudio como lo son el estrés, definido como un estado dinámico mediante el cual el ser humano interactúa con el medio ambiente para mantener el equilibrio necesario para crecer, desarrollarse y desempeñar sus funciones. Y, el conflicto de rol, entendido como el grupo de conductas que se espera de las personas que ocupan una posición y función dentro de un sistema social ${ }^{(12)}$. Al respecto, King afirma que todos tienen una serie de expectativas (superiores, profesionales relacionados, pacientes), las cuales, si difieren de las expectativas propias, generarían conflictos de roles ${ }^{(12,13)}$.

Según lo expuesto esta investigación tuvo como objetivo, evaluar la percepción de estrés del equipo de enfermería de Atención Primaria en Salud de una comuna de la Región del MauleChile relacionándolo con el perfil sociodemográfico, variables laborales y las condiciones ambientales de trabajo de los participantes.

\section{MATERIAL Y MÉTODO}

Estudio transversal, de abordaje cuantitativo ${ }^{(14)}$. Durante los meses de junio y julio de 2017 se censó a 105 trabajadores profesionales de Enfermería y Técnicos de Enfermería (encuestándose un 94,5\% de la población accesible), que se desempeñaban en cuatro Centros de Salud Familiar (CESFAM) de una comuna de la Región del Maule y que cumplían con los criterios de inclusión: no encontrarse con licencias médicas o permiso legal durante la recolección de los datos y aceptación a participar del estudio, por medio de la firma del consentimiento informado. La recolección de datos se realizó a través de la aplicación de un instrumento de forma presencial a los trabajadores, en cada uno de los CESFAM durante su jornada laboral. Inicialmente se explicaron los objetivos 
de la investigación, haciendo entrega de una copia consentimiento informado.

Posteriormente se aplicó instrumento recolector de datos, el cual se dividió en dos partes: a) Antecedentes biosociodemográficos y laborales: Cuestionario elaborado por el equipo investigador, donde se consultó antecedentes personales como sexo, edad, situación de pareja; antecedentes laborales, cargo que desempeña, CESFAM en que trabaja, años de experiencia y condiciones ambientales de trabajo. b) "Job Content Questionnaire" (JCQ): este instrumento es uno de los cuestionarios más utilizados para medir tensión mental o estrés psicológico a nivel mundial ${ }^{(15)}$. Se trata de la versión corta, que incluye tres dimensiones, las cuales incorporan diferentes preguntas o ítems: Demandas psicológicas (5 ítems); Control sobre el trabajo (9 ítems) y Apoyo en el trabajo (8 ítems).

Las posibles categorías respuesta de cada uno de los ítems son: totalmente en desacuerdo (uno), en desacuerdo (dos), de acuerdo (tres) y totalmente de acuerdo (cuatro). La dimensión demandas psicológicas valora la cantidad de trabajo, las exigencias intelectuales y la presión del tiempo de trabajo. La dimensión de control sobre el trabajo valora la posibilidad de tomar decisiones, la creatividad y la aplicación y desarrollo de las propias habilidades, considerando dos factores: Utilización de habilidades y Autoridad de Decisión. Finalmente, la dimensión de apoyo en el trabajo evalúa el apoyo recibido por los compañeros y los superiores ${ }^{(16)}$.
Cabe destacar que el cuestionario JCQ fue validado y adaptado por Juárez en un estudio multicéntrico para México, Colombia y Chile ${ }^{(17)}$ y luego ha sido utilizado en otros estudios a nivel nacional ${ }^{(18-20)}$.

Para el análisis estadístico, en primer lugar, se describieron las variables de perfil sociodemográfico y el nivel de estrés percibido por los trabajadores del equipo de enfermería de Atención Primaria de Salud de la comuna de Linares, utilizando frecuencias relativas y absolutas, promedio, DT y rangos. Además, se relacionaron las variables de nivel de estrés percibido por la población de estudio con variables laborales, con perfil sociodemográfico y finalmente con condiciones ambientales de trabajo, en donde se utilizaron los estadígrafos de Chi cuadrado y Correlación de Pearson. Para el análisis inferencial se consideró un valor $p \leq 0,05$.

Durante todo el proceso de la investigación se consideraron los aspectos éticos considerados por Ezequiel Emanuel, incluyendo confidencialidad, voluntariedad y consentimiento informado ${ }^{(21)}$, y fue aprobado por el Comité Ético-Científico de la Universidad Católica del Maule y el Comité ÉticoCientífico del Servicio de Salud del Maule.

\section{RESULTADOS}

A continuación se presentan los resultados de la investigación, con la intención de responder al objetivo del estudio.

Tabla 1. Variables Personales de los trabajadores del equipo de enfermería de los CESFAM de la comuna de Linares participantes del estudio $(\mathrm{n}=105)$.

\begin{tabular}{|c|c|c|c|}
\hline Características Biosociodemográficas & & $f x$ & $\%$ \\
\hline \multirow{2}{*}{ Sexo } & Hombre & 17 & 16,2 \\
\hline & Mujer & 88 & 83,8 \\
\hline \multirow[t]{6}{*}{ Edad en años (Media: 35,70; DE: 10,24; R: 23-62) } & 20 a 30 años & 39 & 37,1 \\
\hline & 31 a 40 años & 39 & 37,1 \\
\hline & 41 a 50 años & 12 & 11,4 \\
\hline & 51 a 60 años & 12 & 11,4 \\
\hline & Mayor 60 años & 2 & 1,9 \\
\hline & No responde & 1 & 0,95 \\
\hline \multirow{2}{*}{ Situación de Pareja } & Sin Pareja & 21 & 20 \\
\hline & Con Pareja & 84 & 80 \\
\hline \multirow[t]{6}{*}{$\mathrm{N}^{\circ}$ de Hijos (Media: 1,22; DE: 1,07; R: 0-4) } & 0 & 34 & 32,4 \\
\hline & 1 & 28 & 26,6 \\
\hline & 2 & 28 & 26,6 \\
\hline & 3 & 13 & 12,4 \\
\hline & 4 & 1 & 0,95 \\
\hline & No responde & 1 & 0,95 \\
\hline
\end{tabular}


Perfil biosociodemográfico y laboral de la narios se encuentran entre los 20 y 40 años, y en población de estudio

En la Tabla 1 se presentan las características biosociodemográficas de los participantes del estudio; de ellas se destaca, el 74,2\% de los funciorelación al número de hijos, el 53,4\% tiene entre uno y dos hijos.

Respecto a las variables laborales, se evidencia en la Tabla 2 que la mayoría de los funcionarios

Tabla 2. Variables Laborales de los trabajadores participantes del estudio $(n=105)$.

\begin{tabular}{llcc}
\hline Variables & & fx & $\%$ \\
\hline Cargo que desempeña & Enfermera/o & 31 & 29,5 \\
CESFAM al que pertenece & TENS & 74 & 70,5 \\
& CESFAM 1 & 25 & 23,8 \\
& CESFAM 2 & 22 & 21 \\
& CESFAM 3 & 37 & 35,2 \\
Años de experiencia & CESFAM 4 & 14 & 13,3 \\
& CECOSF & 7 & 6,7 \\
& 0 a 10 años & 86 & 81,9 \\
Situación Contractual & 11 a 20 años & 10 & 9,5 \\
& 21 a 30 años & 5 & 4,8 \\
& 31 a 40 años & 4 & 3,8 \\
& Indefinido & 45 & 42,9 \\
& A contrata & 20 & 19 \\
& Honorarios & 30 & 28,6 \\
& Otras & 10 & 9,5 \\
\hline
\end{tabular}

Tabla 3. Condiciones ambientales de trabajo de los trabajadores del equipo de enfermería de los CESFAM de la comuna de Linares participantes del estudio $(n=105)$.

\begin{tabular}{|c|c|c|c|}
\hline Condiciones ambientales de trabajo & & $f x$ & $\%$ \\
\hline \multirow[t]{5}{*}{ Temperatura en el puesto de trabajo } & Muy inadecuada & 9 & 8,6 \\
\hline & Inadecuada & 12 & 11,4 \\
\hline & Intermedia & 27 & 25,7 \\
\hline & Adecuada & 35 & 33,3 \\
\hline & Muy adecuada & 22 & 21 \\
\hline \multirow[t]{5}{*}{ Iluminación en el puesto de trabajo } & Muy inadecuada & 4 & 3,8 \\
\hline & Inadecuada & 4 & 3,8 \\
\hline & Intermedia & 27 & 25,7 \\
\hline & Adecuada & 39 & 37,1 \\
\hline & Muy adecuada & 31 & 29,5 \\
\hline \multirow[t]{5}{*}{ Ruido en el puesto de trabajo } & Muy inadecuada & 4 & 3,8 \\
\hline & Inadecuada & 9 & 8,6 \\
\hline & Intermedia & 30 & 28,6 \\
\hline & Adecuada & 42 & 40 \\
\hline & Muy adecuada & 20 & 19 \\
\hline \multirow[t]{5}{*}{ Espacio en el puesto de trabajo } & Muy inadecuada & 17 & 16,2 \\
\hline & Inadecuada & 21 & 20 \\
\hline & Intermedia & 22 & 21 \\
\hline & Adecuada & 28 & 26,7 \\
\hline & Muy adecuada & 17 & 16,2 \\
\hline \multirow[t]{5}{*}{ Condiciones de higiene en el puesto de trabajo } & Muy inadecuada & 9 & 8,6 \\
\hline & Inadecuada & 9 & 8,6 \\
\hline & Intermedia & 39 & 37,1 \\
\hline & Adecuada & 34 & 32,4 \\
\hline & Muy adecuada & 14 & 13,3 \\
\hline
\end{tabular}


Tabla 4. Descripción de la percepción de las dimensiones de JCQ de los trabajadores participantes del estudio $(n=105)$.

\begin{tabular}{|c|c|c|c|}
\hline Job Content Questionnaire (JCQ) & & $f x$ & $\%$ \\
\hline \multirow[t]{3}{*}{ Demandas Psicológicas } & Media: 29,9; DE: 7,3; R: 12 - 46 & & \\
\hline & Baja demanda & 50 & 47,6 \\
\hline & Alta demanda & 55 & 52,4 \\
\hline \multirow[t]{3}{*}{ Control laboral } & Media: 71,9; DE: 11,5; R: 40 - 94 & & \\
\hline & Bajo control & 40 & 38,1 \\
\hline & Alto control & 65 & 61,9 \\
\hline \multirow[t]{3}{*}{ Apoyo Social } & Media: 26,1; DE: 4,5; R: $16-32$ & & \\
\hline & Bajo apoyo & 32 & 30,5 \\
\hline & Alto apoyo & 73 & 69,5 \\
\hline \multirow[t]{3}{*}{ Inseguridad del empleo } & Media: 5,0; DE: 1,6; R: $3-10$ & & \\
\hline & Baja inseguridad & 54 & 51,4 \\
\hline & Alta inseguridad & 51 & 48,6 \\
\hline \multirow[t]{3}{*}{ Tensión Laboral } & Mínimo: 0; Máximo:1 & & \\
\hline & Sin tensión laboral & 80 & 76,2 \\
\hline & Con tensión laboral & 25 & 23,8 \\
\hline
\end{tabular}

Tabla 5. Relación de las variables biosociodemográficas y laborales con las dimensiones de JCQ.

\begin{tabular}{llllll}
\hline Variables del estudio & $\begin{array}{c}\text { Control } \\
\text { laboral }\end{array}$ & $\begin{array}{c}\text { Demandas } \\
\text { psicológicas }\end{array}$ & $\begin{array}{c}\text { Apoyo } \\
\text { Social }\end{array}$ & $\begin{array}{c}\text { Inseguridad } \\
\text { en el Empleo }\end{array}$ & $\begin{array}{c}\text { Tensión } \\
\text { Mental }\end{array}$ \\
\hline Variables Biosociodemográficas & & & & & \\
Sexo & $-0,122$ & $-0,139$ & $-0,061$ & $-0,063$ & $-0,058$ \\
Edad & 0,02 & $-0,08$ & $-0,03$ & $-0,381^{* *}$ & $-0,076$ \\
$N^{\circ}$ de hijos & $-0,119$ & $-0,026$ & $-0,165$ & $-0,263^{* *}$ & 0,094 \\
\hline Variables Laborales & & & & & \\
Experiencia en años & $-0,013$ & $-0,007$ & 0,016 & $-0,446^{* *}$ & $-0,041$ \\
Cargo & $-0,281^{* *}$ & $-0,007$ & $-0,068$ & 0,038 & 0,166 \\
\hline Variables Ambientales de trabajo & & & & & \\
Temperatura en su puesto & 0,02 & $-0,359^{* *}$ & $0,227^{* *}$ & 0,113 & $-0,220^{*}$ \\
Iluminación en su puesto & 0,087 & $-0,304^{* *}$ & $0,325^{* *}$ & 0,151 & $-0,247^{*}$ \\
Ruido en su puesto & 0,176 & $-0,228^{*}$ & 0,134 & 0,063 & $-0,188$ \\
Espacio en su puesto & $0,234^{*}$ & $-0,324$ & 0,123 & 0,039 & $-0,298^{* *}$ \\
Higiene en su puesto & $0,371^{* *}$ & $-0,198^{*}$ & $0,237^{* *}$ & 0,086 & $-0,254^{* *}$ \\
\hline
\end{tabular}

Para las variables sexo y cargo se utilizó estadigrafo Chi-cuadrado.

Para el resto de las variables se utilizó correlación de Pearson; $p \leq 0,05^{*} ; p \leq 0,01^{* *}$

corresponden a Técnicos en Enfermería (TENS), alcanzando un 70,5\%. En relación a los años de experiencia, el 81,9\% de los participantes lleva entre 0-10 años de experiencia, y, por último, en relación a la situación contractual, el $42 \%$ de los funcionarios reportó tener un contrato indefinido.

Continuando con las condiciones ambientales de trabajo descritas en la Tabla 3, en la variable relacionada con la Temperatura en el puesto de trabajo el 33,3\% del total de la muestra la consideró adecuada; respecto a la iluminación en el puesto de trabajo, se evidenció que el $37,1 \%$ de la población encuestada la considera adecuada. Por otro lado, respecto a la variable ruido en el puesto de trabajo, el 40,0\% del total de los funcionarios lo percibe como adecuada. En relación al Espacio en el puesto de trabajo, el $26,7 \%$ de la muestra lo considera adecuado. Finalmente, respecto a condiciones de higiene en el puesto de trabajo, el $37,1 \%$ de la población las considera intermedia.

En relación a la percepción de las dimensiones de JCQ (Tabla 4), se evidencia que en la 
dimensión de Demandas Psicológicas el 52,4\% de la población presenta una Alta demanda. En relación al Control Laboral, el 61,9\% de la muestra percibe un Alto control. Siguiendo con la dimensión de Apoyo social, se evidenció que el $69,5 \%$ de la población encuestada percibe un Alto apoyo. Respecto a la Inseguridad del empleo, el 51,4\% de los funcionarios reportó una Baja inseguridad. Finalmente, un 23,8\% de los funcionarios/as reporta percibir Tensión mental de origen laboral.

\section{Estrés percibido y su relación con variables sociodemográficas y laborales}

En la Tabla 5 se observan relaciones inversas con significancia estadística entre las variables biosociodemográficas y las dimensiones de JCQ. Se evidencia que a menor edad es mayor la percepción de inseguridad en el empleo por parte de los trabajadores, lo que se repite con el número de hijos, es decir, a menor número de hijos es mayor la percepción de inseguridad laboral. Además, se observa que existe relación entre las dimensiones de JCQ y las variables laborales.

Por otra parte, se observan relaciones negativas y significativas entre las variables ambientales de trabajo y las dimensiones de JCQ. De modo que, a menor satisfacción en cuanto al espacio e higiene en su puesto de trabajo, se percibe un menor control laboral. También se destaca que la percepción de insatisfacción en las variables ambientales aumenta las demandas psicológicas. Es decir que, al existir una insatisfacción en las variables ambientales, aumenta la tensión mental. Finalmente, se observa que, a mayor satisfacción con la temperatura, iluminación e higiene en su puesto, existirá una mayor percepción de Apoyo social. Cabe destacar que no existen variables ambientales que se relacionen significativamente con la variable Inseguridad en el empleo.

\section{DISCUSIÓN}

En primer lugar, respecto de las demandas psicológicas, se evidenció que más de la mitad de los trabajadores participantes $(52,4 \%)$ las reportaron como altas. Este resultado difiere con lo postulado por Klein et. al., ${ }^{(22)}$ en el área intrahospitalaria, en donde un $22,4 \%$ de los trabajadores sanitarios percibieron altas demandas. Según Karasek y
Theorell, se entiende por demandas psicológicas a las exigencias psicológicas que el trabajo implica para la persona. Básicamente hacen referencia a cuánto se trabaja: cantidad o volumen de trabajo, presión de tiempo, nivel de atención, interrupciones imprevistas; por lo tanto, no se circunscriben al trabajo intelectual, sino a cualquier tipo de tarea ${ }^{(10)}$. En base a esto, el equipo investigador postula que el resultado obtenido era esperable dado el contexto de trabajo del grupo de estudio. El equipo de enfermería de atención primaria debe desarrollar múltiples tareas, tales como atender gran cantidad de personas durante el día, lidiar con interrupciones, además de cumplir con los horarios estipulados para cada control ${ }^{(23-24)}$.

En la dimensión inseguridad en el empleo, un alto porcentaje $(48,6 \%)$ percibió alta inseguridad; resultado que coincide con el obtenido en un estudio realizado en México y Chile ${ }^{(25)}$, en donde la inseguridad en el empleo se reportó como un problema de creciente atención. Expertos concluyen que la inseguridad laboral es uno de los estresores más nocivos que los trabajadores deben afrontar en la realidad actual ${ }^{(26)}$, ya que genera incertidumbre que acompaña a la baja certeza de poder conservar el empleo ${ }^{(25)}$. Se postula que esta percepción se pudiera explicar desde la situación contractual que tienen la mayor parte de los trabajadores del equipo de enfermería de este estudio, lo que genera mayor incertidumbre respecto a su estabilidad laboral.

Respecto a la dimensión control laboral, un 38,1 \% reporta percepción de bajo control, estos resultados coinciden con los expuestos por otros investigadores ${ }^{(27)}$, quienes reportaron también una percepción de bajo control. Se entiende por control al cómo se trabaja, y tiene dos componentes: la autonomía y el desarrollo de habilidades. La autonomía es la posibilidad que tiene la persona de influenciar decisiones relacionadas con su trabajo, de controlar sus propias actividades. El desarrollo de habilidades hace referencia al grado en que el trabajo permite a la persona desarrollar sus propias capacidades: aprendizaje, creatividad, trabajo variado. Según Karasek y Theorell se trata de las oportunidades o recursos que la organización proporciona a la persona para moderar o tomar decisiones sobre las demandas en la planificación y ejecución del trabajo ${ }^{(10)}$.

Por otro lado, en la dimensión apoyo social, un 
$30,5 \%$ percibe bajo apoyo social. Según Karasek y Theorell ${ }^{(10)}$, cuando existe una alta demanda y un bajo control (cuadrante de alta tensión) la función del apoyo social es incrementar una habilidad para hacer frente al estrés, por lo que resulta un moderador del efecto que produce el estrés o tensión mental en la salud de las personas. Lo postulado por los expertos se comprueba en que el apoyo social influye en la disminución en los niveles de agotamiento emocional, despersonalización y baja realización personal ${ }^{(28)}$. Es por lo anterior que el equipo investigador afirma que este importante porcentaje de trabajadores, al tener un bajo apoyo social, una alta demanda y ausencia de posibilidad de influir en las decisiones, o no encontrarse en posición de ejercer influencia en aspectos importantes de sus condiciones de trabajo y de poder adaptarlas, pudieran estar expuestos a un mayor riesgo de enfermedades tales como enfermedades cardiovasculares, procesos alérgicos, trastornos músculo-esqueléticos cervicales y de miembro superior, ansiedad ${ }^{(29)}$.

Finalmente, respecto a la dimensión tensión laboral se evidenció que un 23,8\% reporta tensión o estrés psicológico al realizar su trabajo. Se comparan estos resultados con lo propuesto por el modelo de demanda control y apoyo de Karasek y Theorell ${ }^{(10)}$ y corrobora su postulado al identificar que los participantes que perciben tensión laboral son también los que perciben altas demandas, bajo control y bajo apoyo social. Lo anterior genera estrés psicológico, aumentando el proceso de desgaste corporal y el riesgo de tensión psicológica, así también la enfermedad. Se postula que, si este $23,8 \%$ de tensión mental se mantiene, los trabajadores no lograrán hacer frente a los efectos que el estrés provoque en su salud, se enfermarán, aumentando el ausentismo laboral lo que generará problemas en la gestión de recursos humanos, por lo que se abre una brecha investigativa.

Al relacionar la dimensión Control laboral (autonomía y el desarrollo de habilidades), y la variable Cargo que desempeña, se expone que son los Técnicos de Enfermería de Nivel Superior (TENS) quienes perciben un menor control, lo cual probablemente se explica por la autonomía "relativa" que tienen en el desempeño de sus funciones. Se agrega, además, que la mirada de los enfermeros/as es diferente ya que estos cuentan con un rol claramente definido por el código sanitario chileno, el que aporta una mayor autonomía de su trabajo, en el manejo de habilidades duras y mayores conocimientos ${ }^{(30)}$. En base a lo anterior se postula que se podría generar un conflicto de rol, tal como lo plantea King ${ }^{(12)}$.

Por otra parte, al relacionar el Control laboral con la higiene percibida en el lugar de trabajo, se plantea que a menor satisfacción respecto a la higiene en su puesto de trabajo es menor la percepción de Control laboral. Esto puede ser explicado desde una situación de menoscabo en el ambiente laboral, lo que pudiera afectar el desempeño óptimo del trabajador, sobre todo aquellos que necesitan de un área limpia para ejecutar sus funciones, como por ejemplo curaciones, toma de muestras, administración de vacunas, esterilización, etc. Lo que coincide con otro estudio, donde se afirma que un déficit de higiene en el lugar de trabajo deteriora la comunicación, desmotiva y dificulta el cumplimiento de los objetivos establecidos ${ }^{(31)}$.

En cuanto a la relación entre Control laboral y el Espacio en su puesto de trabajo, se expone que el desempeñarse en un espacio reducido afecta negativamente la percepción de Control laboral. Por ejemplo, en situaciones en donde se cuenta con un tiempo acotado para atender a gran cantidad de usuarios en un box pequeño, caso de la toma de muestras. Sumando al uso de un mismo box para varias funciones, lo que entorpece el correcto desempeño laboral. Por su parte, un experto del Instituto de Salud Pública de Chile (ISPCH) expone que un espacio reducido del lugar de trabajo podría provocar dificultad para pensar lógicamente y/o tomar decisiones, entre otros ${ }^{(32)}$.

Siguiendo con la dimensión Demandas psicológicas, se encontraron relaciones significativas con las variables ambientales. Se expone que la insatisfacción de las variables del ambiente de trabajo aumenta la percepción de altas demandas psicológicas, ya que pudieran provocar falta de concentración, desánimo, sueño, fatiga, lo que finalmente interfiere en el desempeño laboral. Expertos señalan que el ruido en el puesto de trabajo disminuye la concentración, la efectividad, la productividad, provoca irritabilidad y estados neuróticos histéricos, además señala que la temperatura reduce la destreza manual, aumenta el tiempo de duración de las tareas asignadas, por lo tanto, provocaría insatisfacción por el trabajo realizado, menor compromiso y cansancio ${ }^{(31-32)}$.

En la dimensión Apoyo social se evidenciaron 
relaciones negativas significativas con las variables temperatura, iluminación e higiene. Así, se muestra que, a menor satisfacción de cualquiera de las variables mencionadas anteriormente en su puesto de trabajo, el trabajador/a percibe bajo apoyo social. Esta situación podría generar estados de desánimo y tensión mental ${ }^{(10)} y$, por consiguiente, afectaría a las relaciones sociales en el lugar de trabajo. King expone que la relación enfermera-paciente es un tipo de sistema interpersonal, definido como un proceso de interacción humana entre ambos, donde se identifican problemas, fijan objetivos, exploran los medios y se ponen de acuerdo para alcanzar los objetivos propuestos. Por lo tanto, la relación es un proceso de acción-reacción-interaccióntransacción ${ }^{(12)}$. Cabe destacar que este tipo de interacción es aplicable a cualquier tipo de relación, tal como en este caso, donde se aplica a la relación que se establece entre el equipo de enfermería y el entorno, específicamente las condiciones ambientales que se detallaron anteriormente, las cuales, si son percibidas de manera desfavorables interferirán en las relaciones que se establezcan entre los trabajadores del equipo de enfermería, por una parte, y el equipo con los usuarios, por otra.

La dimensión Inseguridad en el empleo presentó relaciones significativas con las variables edad, número de hijos y años de experiencia. Se señala que estas tres variables se relacionan entre sí, es decir, a menor edad de los funcionarios, tendrán una menor experiencia en el trabajo y menor número de hijos. Esto último se sustenta desde expertos, quienes fundamentan que los jóvenes privilegiarían tener una mayor estabilidad laboral para adquirir inmuebles, vehículo, entre otros bienes, que pensar en la posibilidad de tener o no hijos; dependen de la estabilidad económica ${ }^{(33)}$.

Por otra parte, cabe señalar que el campo laboral de Enfermería a nivel mundial ha ido disminuyendo considerablemente, puesto que no hay cargos disponibles ${ }^{(23,34)}$, lo que se relacionaría directamente con la saturación que presentan las carreras de Enfermería y técnicos en Enfermería, posicionándolas en el quinto y tercer lugar a nivel nacional, respectivamente; todo lo anterior haría más difícil conseguir un contrato de tipo indefinido que otorgue mayor estabilidad laboral. Sumando a lo expuesto, el tener alta inseguridad en el empleo aumentará la presión para realizar sus actividades de manera óptima, y al existir poca experiencia laboral, la ejecución de sus funciones se puede ver influenciada de forma negativa, llevándolos a cometer errores o no rendir de forma eficiente y eficaz, generando por consiguiente conflicto de rol $^{(12)}$. Cabe destacar que en el caso de aquellos funcionarios que cuentan con una larga trayectoria en el sistema sanitario y llevan un corto tiempo desempeñándose en su lugar actual de trabajo, el conflicto de rol se presentaría de igual forma, debido al cambiante contexto laboral, es decir, cambios en el equipo de trabajo, modificaciones ambientales y de carga laboral. Todo esto también afectaría la consecución de objetivos ${ }^{(12,13)}$, ya que al existir conflicto de rol en algunos de los actores del sistema que interactúan, se generará estrés, lo cual impacta de forma negativa e interfiere en el logro de metas establecidas.

Finalmente, en relación a la dimensión Tensión mental se evidenció que las variables temperatura, iluminación, espacio e higiene resultaron estadísticamente significativas. Cabe destacar que todas las dimensiones de JCQ se correlacionan, a lo menos, con una variable de las condiciones ambientales de trabajo, excepto la dimensión de inseguridad en el empleo. Esto visualiza que el entorno estructural efectivamente se relaciona con la percepción de Tensión mental, tal como indica King al afirmar que la "interacción” es: un proceso de percepción y comunicación entre una persona y otra o entre una persona y el entorno ${ }^{(12)}$, lo cual lleva a considerar como elemento esencial la relación del entorno sobre la persona, y la relación de ello con la percepción de tensión mental, es decir, si se percibe una menor satisfacción del ambiente de trabajo, la percepción que resultará entre la interacción que se genera entre este entorno y los trabajadores será negativa, lo cual afectaría el proceso de consecución de metas ${ }^{(13)}$ y, finalmente, generará tensión mental de origen laboral en el equipo de enfermería.

\section{CONCLUSIÓN}

Se concluye que el 23,8\% de los trabajadores del equipo de Atención Primaria en Salud (APS) de la comuna de Linares perciben Tensión mental al desempeñar sus labores. Si bien en este grupo de estudio no se presentó relación significativa con las variables biosociodemográficas ni con las variables laborales, sí se estableció una relación 
estadísticamente significativa con las variables ambientales de trabajo, lo que da paso a que en el futuro inmediato los empleadores mejoren las condiciones en el trabajo, y que los estamentos encargados del ámbito laboral a nivel nacional enfaticen en la fiscalización y supervisión de esta variable, con el fin de reducir el porcentaje de trabajadores susceptibles a sufrir enfermedades a causa de la tensión mental, de lo contrario, podría provocar alteraciones en su estado de salud.

Se postula que es necesario seguir investigando respecto a los factores psicosociales en contextos laborales, como el estrés en trabajadores de Atención Primaria en Salud, con el propósito de aportar al fortalecimiento de la calidad de vida laboral de estos trabajadores.

Como limitación del estudio, se indica la dificultad de generalizar los resultados de la investigación a otras latitudes o contextos de trabajo.

\section{REFERENCIAS}

1. Leka S, Griffiths A, Cox T. La organización del trabajo y el estrés: estrategias sistemáticas de la solución de problemas para empleadores, personal directivo y representantes sindicales [Internet]. Ginebra: Organización Mundial de la Salud; 2004 [citado 19 May 2018]. 37 p. Disponible en: http://www.who.int/iris/handle/10665/42756

2. Petreanu V, Iordache R, Seracin M. Assessment of Work Stress Influence on Work Productivity in Romanian Companies. Procedia Soc Behav Sci. [Internet]. 2013 Oct 10 [cited 2018 May 19]; 92: 420-425. Available from: https://doi. org/10.1016/j.sbspro.2013.08.695

3. Junne F, Michaelis M, Rothermund E, Stuber F, Gündel H, Zipfel S, et al. The Role of Work-Related Factors in the Development of Psychological Distress and Associated Mental Disorders: Differential Views of Human Resource Managers, Occupational Physicians, Primary Care Physicians and Psychotherapists in Germany. Int. J. Environ. Res. Public Health. [Internet]. 2018 Mar 20 [cited 2018 May 19]; 15(3): 559. Available from: https://www.ncbi.nlm. nih.gov/pubmed/29558427

4. Miranda M. Licencias laborales por salud mental suben y llegan al $48 \%$ de permisos en la AChs. Diario La Tercera. [Internet]. 2014 Nov 11 [citado 19 May 2018]. Disponible en: http://www2. latercera.com/noticia/licencias-laborales-porsalud-mental-suben-y-llegan-al-48-de-permisosen-la-achs/
5. CremadesJ, MaciáL, LópezM, PedrazA, González V. A new contribution to the classification of stressors affecting nursing professionals. Rev. Latino-Am. Enfermagem. [Internet]. 2017 May 22 [cited 2018 Jun 15]; 25: e2895. Available from: http://dx.doi.org/10.1590/1518-8345.1240.2895

6. Consejo Internacional de Enfermería. Las enfermeras, una fuerza para el cambio: eficaces en cuidados, eficientes en costos. [Internet]. Ginebra: CIE; 2015 [citado 19 May 2018]. 70 p. Disponible en: http://files.sld.cu/socuenf/files/2015/04/carpetadel-die_2015_sp1.pdf

7. Organización Internacional del Trabajo. Estrés en el trabajo: Un reto colectivo. Servicio de Administración del Trabajo, Inspección del Trabajo y Seguridad y Salud en el Trabajo. [Internet]. Ginebra: OIT; 2016 [citado 19 May 2018]. 68 p. Disponible en: http://www.ilo.org/wcmsp5/groups/ public/---ed_protect/---protrav/---safework/ documents/publication/wcms_466549.pdf

8. Rodrigues C, Salvador P, Assis Y, Gomes A, Bezerril M, Santos V. Stress among nursing team members. J Nurs UFPE. [Internet]. 2017 Feb [cited 2018 May 19]; 11(2): 601- 608. Available from: https:// periodicos.ufpe.br/revistas/revistaenfermagem/ article/view/11979

9. Locihová $H$, Axmann $K$, Padyšáková $H$, Pončíková V. Perception of intensive care stressors by patients, nurses and family. Cent Eur J Nurs Midw. [Internet]. 2018 [2019 Jan 19]; 9(1): 758766. Available from: https://periodika.osu.cz/ojs/ index.php/cejnm/article/view/105

10. Karasek R, Theorell T. Healthy work. Stress, productivity, and the reconstruction of working life. New York: Basic Books; 1990. 475 p.

11. Bucci N, Luna M. Contrastación entre los modelos de estudio del estrés como soporte para la evaluación de los riesgos psicosociales en el trabajo. Revista digital de investigación y postgrado [Internet]. 2012 [citado 19 Ene 2019]; 2(1): 21-38. Disponible en: https://dialnet. unirioja.es/servlet/articulo?codigo $=3908567$

12. King I. A theory for nursing: systems, concepts, process. New York: John Wiley y Sons; 1981. $181 \mathrm{p}$.

13. King I. The theory of goal attainment. In: Frey MA, Sieloff CL (eds.). Advancing King's systems framework and theory of nursing. Thousand Oaks, CA: Sage; 1995. p. 23-32.

14. Rouquayrol MZ, Gurgel M. Rouquayrol: Epidemiologia e Saúde. Brasil: Med Book; 2018. $744 \mathrm{p}$.

15. Karasek R, Brisson C, Kawakami N, Houtman I, Bongers P, Amick B. The Job Content Questionnaire (JCQ): an instrument for internationally comparative assessments of 
psychosocial job characteristics. J Occup Health Psychol [Internet]. 1998 Oct [citado 19 May 2018]; 3(4): 322-355. Available from: https:// www.ncbi.nlm.nih.gov/pubmed/9805280

16. Escribà-Agüir V, Más Pons R, Flores Reus E. Validación del Job Content Questionnaire en personal de enfermería hospitalario. Gac Sanit. [Internet]. 2001 Mar [citado 19 May 2018]; 15(2): 91-190. Disponible en: http://www.gacetasanitaria.org/ es-validacion-del-job-content-questionnairearticulo-resumen-S0213911101715336

17. Juárez-García A, Vera A, Gómez V, Canepa C, Schnall M. El Modelo Demanda/Control y la salud mental en profesionales de la salud: un estudio en tres países latinoamericanos. Foro de las Américas de Investigación sobre Factores Psicosociales. 2008:1-11.

18. Chiang $M$, Gómez N, Sigoña M. Factores psicosociales, estrés y su relación con el desempeño: comparación entre centros de salud. Salud trab [Internet]. 2013 Jul-Dic [citado 19 May 2018]; 21(2): 111-128. Disponible en: http:// www.redalyc.org/pdf/3758/375839307002.pdf

19. Juárez A, Vera A, Merino C, Gómez V, Feldman L, Hernández E. Demanda/Control y la Salud Mental en Profesionales de la Salud: Un Estudio en Seis Países Latinoamericanos. Información psicológica. [Internet]. 2014 [citado 19 May 2018]; 108: 2-18. Disponible en: doi: dx.medra. org/10.14635/IPSIC.2014.108.1

20. Chandía F, Vidal K, Chiang M. Relación entre estrés laboral y adherencia a la dieta mediterránea (DM) en funcionarios de una institución de educación superior, Chile. Cienc Trab. [Internet]. 2016 Ene-Abr [citado 19 May 2018]; 18(55): 58-67. Disponible en: https://scielo.conicyt.cl/scielo.php?script=sci_ arttext\&pid=S0718-24492016000100010

21. Emanuel EJ, Wendler D, Grandy C. What make clinical research ethical?. JAMA. [Internet]. 2000 May [cited 2019 May 19]; 283(20): 2701-2711. Available from: https://www.ncbi.nlm.nih.gov/ pubmed/10819955

22. Klein J, GrosseFrie K, Blum K, Von Dem Knesebeck O. Psychosocial stress at work and perceived quality of care among clinicians in surgery. BMC Health Serv Res. [Internet]. 2011 May 20 [cited 2018 May 19]; 11: 109. Available from: https://www.ncbi. nlm.nih.gov/pubmed/21599882

23. Seguel-Palma F, Valenzuela-Suazo S, SanhuezaAlvarado O. El trabajo del profesional de enfermería: revisión de la literatura. Cienc. enferm. [Internet]. 2015 Ago [citado 19 May 2018]; 21(2): 11-20. Disponible en: https:// scielo.conicyt.cl/scielo.php?script $=$ sci arttext\&pid=S0717-95532015000200002
24. Pires DEP, Machado RR, Soratto J, Scherer MA, Gonçalves ASR, Trindade LL. Cargas de trabajo de enfermería en la salud de la familia: implicaciones para el acceso universal. Rev Lat Am Enfermagem [Internet]. 2016 [citado 19 May 2018]; 24: e2682. Disponible en: http://dx.doi. org/10.1590/1518-8345.0992.2682

25. Juárez A. Factores psicosociales laborales relacionados con la tensión arterial y síntomas cardiovasculares en personal de enfermería en México. Salud Pública Méx. [Internet]. 2007 [citado 19 May 2018]; 49(2): 109-117. Disponible en: http://www.scielo.org.mx/scielo.php?script=sci arttext\&pid=S0036-36342007000200006

26. Sora B, Caballer A, Peiró J. La inseguridad laboral y sus consecuencias en un contexto de crisis económica. Papeles del Psicólogo. [Internet]. 2014 [citado 19 May 2018]; 35(1): 15-21. Disponible en: http://www.papelesdelpsicologo. es/pdf/2317.pdf

27. Wang LJ, Chen CK, Hsu SC, Lee SY, Wang CS, Yeh WY. Active Job, Healthy Job? Occupational Stress and Depression among Hospital Physicians in Taiwan. Ind Health. [Internet]. 2011 [cited 201 May 19]; 49(2): 173-184. Available from: https:// www.ncbi.nlm.nih.gov/pubmed/21173533

28. Portero de la Cruz S, Abellán M. Desgaste profesional, estrés y satisfacción laboral del personal de enfermería en un hospital universitario. Rev Lat Am Enfermagem [Internet]. 2015 May-Jun [citado 19 May 2018]; 23(3): 543-552. Disponible en: http://dx.doi.org/10.1590/01041169.0284 .2586

29. Gómez-Rojas P, Hernández-Guerrero J, MéndezCampos M. Factores de Riesgo Psicosocial y Satisfacción Laboral en una Empresa Chilena del Área de la Minería. Cienc Trab. [Internet]. 2014 Abr [citado 19 May 2018]; 16(49): 9-16. Disponible en: http://dx.doi.org/10.4067/S071824492014000100003

30. Ceballos-Vásquez P, Jara-Rojas A, StiepovichBertoni J, Aguilera-Rojas P, Vílchez-Barboza V. La gestión del cuidado: una función social y legal de la enfermería chilena. Rev. Enfermería Actual en Costa Rica. [Internet]. 2015 Jul-Dic [citado 19 May 2018]; 29: 1-12. Disponible en: https:// revistas.ucr.ac.cr/index.php/enfermeria/article/ view/19733/19910

31. Santoyo-Telles F, Murguía-Pérez D, LópezEspinoza A, Santoyo-Teyes E. Comportamiento y organización. Implementación del sistema de gestión de la calidad 5 S'S. Divers: Perspect. Psicol. [Internet]. 2013 Jul-Dic [citado 19 May 2018]; 9(2): 361-371. Disponible en: http://www.scielo. org.co/scielo.php?script=sci_arttext $\&$ pid=S179499982013000200010\&lng=en\&tlng=es 
32. Instituto de Salud Pública de Chile. Guía de Ergonomía: Identificación y control de factores de riesgo en el trabajo de oficina y el uso de computador. [Internet]. Chile: ISP; 2016 [citado 19 May 2018]. 66 p. Disponible en: http://www.ispch.cl/sites/default/files/D031PR-500-02-001\%20Guia\%20ergonomia\%20 trabajo\%20oficina\%20uso\%20PC.pdf

33. Benítez-Barajas L. Proyectos de vida en parejas de jóvenes adultas y adultos profesionales de Bogotá.
Trab. Soc. [Internet]. 2013 [citado 19 May 2018]; 15: 59-85. Disponible en: https://revistas.unal. edu.co/index.php/tsocial/article/view/42578

34. Sindicato de Enfermería. Análisis del empleo en enfermería. Oferta y demanda de profesionales de enfermería. [Internet]. España: SATSE; 2016 [citado 19 May 2018]. 11 p. Disponible en: https://www.actasanitaria.com/wp-content/ uploads/2016/03/analisis-del-empleo-enenfermeria.pdf 